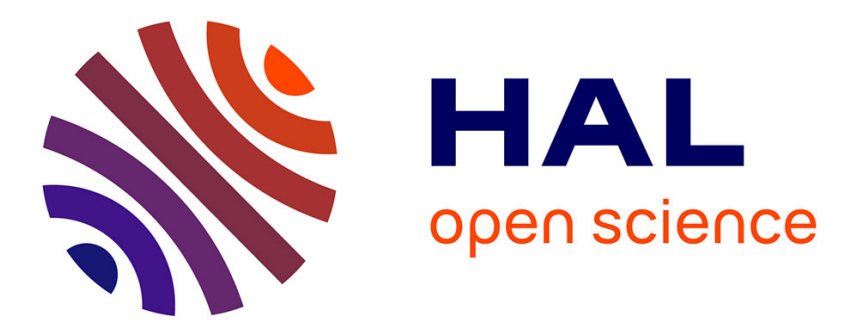

\title{
Mitochondrial diversity and phylogeographic analysis of Pediculus humanus reveals a new Amazonian clade "F"
}

Nadia Amanzougaghene, Florence Fenollar, Bernard Davoust, Félix Djossou, Muhammad Ashfaq, Idir Bitam, Didier Raoult, Oleg Mediannikov

\section{- To cite this version:}

Nadia Amanzougaghene, Florence Fenollar, Bernard Davoust, Félix Djossou, Muhammad Ashfaq, et al. Mitochondrial diversity and phylogeographic analysis of Pediculus humanus reveals a new Amazonian clade "F". Infection, Genetics and Evolution, 2019, 70, pp.1-8. 10.1016/j.meegid.2019.02.006 . hal-02243787

\section{HAL Id: hal-02243787 \\ https://hal-amu.archives-ouvertes.fr/hal-02243787}

Submitted on 22 Oct 2021

HAL is a multi-disciplinary open access archive for the deposit and dissemination of scientific research documents, whether they are published or not. The documents may come from teaching and research institutions in France or abroad, or from public or private research centers.
L'archive ouverte pluridisciplinaire HAL, est destinée au dépôt et à la diffusion de documents scientifiques de niveau recherche, publiés ou non, émanant des établissements d'enseignement et de recherche français ou étrangers, des laboratoires publics ou privés.

\section{(ㄷ)(1) $\$$}

Distributed under a Creative Commons Attribution - NonCommerciall 4.0 International 


\section{Mitochondrial diversity and phylogeographic analysis of Pediculus}

3 Nadia Amanzougaghene ${ }^{1}$, Florence Fenollar ${ }^{2}$, Bernard Davoust ${ }^{1}$, Félix Djossou ${ }^{3}$,

4 Muhammad Ashfaq $^{4}$, Idir Bitam ${ }^{2,5}$, Didier Raoult ${ }^{*}$, Oleg Mediannikov $^{1 *}$

5

6 '1 Aix Marseille Univ, IRD, APHM, MEPHI, IHU-Méditerranée Infection, Marseille, France

$7 \quad{ }^{2}$ Aix Marseille Univ, IRD, APHM, SSA, VITROME, IHU-Méditerranée Infection, Marseille,

8 France

$9{ }^{3}$ Service de maladies Infectieuses et Tropicales, Centre Hospitalier de Cayenne, 97306

10 Cayenne Cedex, Guyane Française, France

$11{ }^{4}$ Centre for Biodiversity Genomics, Biodiversity Institute of Ontario, University of Guelph,

12 Guelph, ON, Canada

13 Ecole Supérieure des Sciences de l'Aliment et des Industries Agro-Alimentaires, Algiers,

14 Algeria

$15 *$ Corresponding authors :

16 Dr. Oleg MEDIANNIKOV

17 Address: MEPHI, IRD, APHM, IHU-Méditerranée Infection, 19-21 Boulevard Jean Moulin, 1813385 Marseille Cedex 05

19 Tel: +33 (0)4 137324 01, Fax: +33 (0)4 137324 02, E-mail: olegusss1@ gmail.com

20 Prof. Didier RAOULT

21 Address: MEPHI, IRD, APHM, IHU-Méditerranée Infection, 19-21 Boulevard Jean Moulin,

2213385 Marseille Cedex 05

23 Tel: +33 (0)4 137324 01, Fax: +33 (0)4 137324 02, E-mail: didier.raoult@ gmail.com

24 Word abstract count: 250

25 Word text count: $\mathbf{3 , 3 2 5}$ 


\section{Abstract}

Pediculus humanus is an obligate and highly intimate bloodsucking insect parasite of

28 humans that has two ecotypes, head louse and body louse. This study analyzed genetic

29 diversity at three mitochondrial genes (cytochrome $b$ [cytb], cytochrome oxidase subunit 1

30 [coxl] and 12S ribosomal RNA [12S]) in 98 head lice collected from an isolated Native

31 American population from the Wayampi community in Trois-Sauts, French Guiana. These

32 results are integrated with all prior data of P. humanus (1402 cytb, 743 cox 1 and $34412 S)$

33 from other parts of the world. The phylogenetic analysis revealed six highly divergent and

34 well-supported monophyletic clades. Five clades corresponded to the previously recognized

35 mitochondrial clades A, D, B, C and E, while the sixth (clade F) was novel, as it exhibited

$365.4 \%, 3.7 \%$ and $3.6 \%$ divergence at cytb, coxl and $12 \mathrm{~S}$, respectively, from its nearest

37 neighbor clade B. Interestingly, the clade F has only been recovered in a few lice sequences

38 from Mexico and Argentina, while it was the most common lineage in the Amazonian lice,

39 which hints its association with the Native American region. Furthermore, Pediculus

40 mjobergi, a New World monkeys' louse, which is thought to be transmitted to monkeys from

41 the first humans that had reached the American continent thousands of years ago, also

42 belonged to this clade, suggesting that this louse may not be a separate species but an

43 evolutionary lineage of $P$. humanus.

$44 \quad$ The discovery of new Amazonian clade F with the recovery of additional haplotypes

45 within each of the five clades demonstrates that the levels of genetic diversity in $P$. humanus

46 are higher than previously thought.

49 Keywords: Pediculus humanus; phylogeography; genetic diversity; Clade F; Amazonia 50 


\section{Introduction}

Sucking lice (Phthiraptera: Anoplura) are obligate blood-feeding ectoparasites of

53 placental mammals that have coevolved with their hosts for more than 65 million years

54 (Durden and Musser, 1994; Light et al., 2010). Humans are parasitized by two species of 55 sucking lice, the pubic louse (Pthirus pubis) and head/body lice (Pediculus humanus) (Reed 56 et al., 2004). The association between the $P$. humanus and its human host can be traced back 57 to at least 6 million years ago (MYa) to a common ancestor of humans and chimpanzees 58 (Reed et al., 2004). P. humanus includes two ecotypes, head lice (P. h. capitis) and body lice 59 (P. h. humanus), that are morphologically and biologically almost similar but ecologically 60 distinct (Reed et al., 2004; Veracx and Raoult, 2012). Head lice are confined to the scalp and

61 feed on human blood every 4-6 hours (Veracx and Raoult, 2012). Body lice live in clothing 62 and feed less frequently but take larger blood meals than head lice (Veracx and Raoult, 63 2012). Aside from their role as pests (Chosidow, 2000), body lice are the main vectors of 64 serious human pathogens; Rickettsia prowazekii (the causative agent of epidemic typhus), 65 Bartonella quintana (trench fever), Borrelia recurrentis (relapsing fever) and probably 66 Yersinia pestis (plague) (Raoult, 2016; Raoult and Roux, 1999). It was once believed that 67 only body lice could transmit disease. However, recently several combined epidemiological 68 and laboratory studies have strongly implicated head lice as a vector of infectious agents, 69 although its vectorial capacity is lower as compared to body lice (Amanzougaghene et al., 70 2017, 2016a; Angelakis et al., 2011; Diatta et al., 2014; Goldberger and Anderson, 1912; Kim 71 et al., 2017; Murray and Torrey, 1975; Sangaré et al., 2014). The genetic diversity of human lice has been extensively studied using mitochondrial 73 genes (mainly cytochrome b [cytb] and cytochrome oxidase subunit 1 [coxl] genes) revealing 74 the presence of five highly divergent mitochondrial clades (A, D, B, C and E) 75 (Amanzougaghene et al., 2016b; Ascunce et al., 2013; Ashfaq et al., 2015; Drali et al., 2015; 
76 Kittler et al., 2003; Reed et al., 2004). In addition to this inter-clade diversity, human lice also 77 present intra-clade diversity, illustrated by several distinct haplotypes for each clade 78 (Amanzougaghene et al., 2016a; Ascunce et al., 2013; Light et al., 2008). Body lice belong to 79 clades $\mathrm{A}$ and $\mathrm{D}$, while head lice encompass the full genetic diversity of clades 80 (Amanzougaghene et al., 2016a; Drali et al., 2016; Light et al., 2008). Clade A is the most 81 common and widely distributed across all continents, whereas the other clades are 82 geographically restricted (Amanzougaghene et al., 2016b; Ascunce et al., 2013; Light et al., 83 2008; Raoult et al., 2008). Clade D is restricted to Africa and is found in the Democratic 84 Republic of Congo (DR Congo), the Republic of Congo (Congo-Brazzaville), Ethiopia and 85 Zimbabwe (Amanzougaghene et al., 2016a; Drali et al., 2015). Clade B is found in America, 86 Europe, Australia, Algeria, South Africa, Saudi Arabia and has recently been found among 87 the remains of Israeli head lice, which date back about 2,000 years (Al-Shahrani et al., 2017; 88 Amanzougaghene et al., 2016b; Ascunce et al., 2013; Ashfaq et al., 2015; Boutellis et al., 89 2015; Light et al., 2008; Raoult et al., 2008). Clade C has been found in some African and 90 Asian countries including Ethiopia, Congo-Brazzaville, Nepal, Pakistan and Thailand 91 (Amanzougaghene et al., 2016a; Ashfaq et al., 2015; Kittler et al., 2003; Raoult et al., 2008; 92 Sunantaraporn et al., 2015). Finally, clade E appears to be specific to West African lice, 93 including Senegal and Mali, where it is highly prevalent, and has recently been identified in 94 the head lice from Nigerian refugees arriving in Algeria and from migrant communities living 95 in Bobigny, France (Amanzougaghene et al., 2017; Candy et al., 2018; Louni et al., 2018). Lice are highly host specific and fast-evolving parasites that have evolved in tandem 97 with their primate hosts for thousands of years (Light et al., 2008; Reed et al., 2007). 98 Previous studies have shown that the time of divergence among lice clades (around 2 MYa) 99 was far anterior to the time of modern human divergence, about 200,000 years ago (Light et 100 al., 2008; Tishkoff et al., 2009). Previous reports have also suggested that different lice clades 
101

have evolved on other archaic hominids, likely those known from 2.3 to $0.03 \mathrm{MYa}$ (such as Homo erectus, Neandertal and Denisovan), and have only switched to modern humans during the recent period of overlaps (Ashfaq et al., 2015; Reed et al., 2004). Moreover, the presence of highly divergent clades and their geographical isolation can yield important information regarding the evolutionary history of the lice as well as their human hosts (Ascunce et al., 2013; Light et al., 2008). Therefore, a more detailed analysis of genetic diversity in $P$. humanus and current distributions of its major clades will provide a more detailed picture of evolutionary pattern of this parasite and will clarify additional events in human evolution.

In the present study, we obtained and analyzed head lice collected from an isolated Native American population from the Wayampi community in Trois-Sauts, French Guiana. These results are integrated with all prior mitochondrial data from over the world to expand perspectives on the number, distributions and diversification rates of clades of Pediculus lice.

\section{Materials and methods}

\section{1 Ethical approval}

The Amazonian head lice were collected from infested Amerindians after obtaining their verbal consent or that of their legal representatives in the case of children, because most subjects were illiterate. Local authorities approved and were present at the lice collection. The study was validated by the ethics committee of the Institut Fédératif de Recherche 48, Faculty of Medicine, Marseille, France, under agreement12-017.

\section{2 Louse samples}

The Amazonian head louse specimens were recovered in 2013 from Amerindians of the Wayampi community living in Trois-Sauts $\left(2^{\circ} 15^{\prime} 0^{\prime \prime} \mathrm{N}\right.$ and $\left.52^{\circ} 52^{\prime} 60^{\prime \prime} \mathrm{W}, 122 \mathrm{~m}\right)$, a remote and isolated village in French Guyana. A total of 98 head lice were recovered from 22 individuals. No body lice were found during the examination. Collected lice were then preserved in $70 \%$ ethanol before being sent to our laboratory in Marseille (France). All the 
samples were photographed with a camera (Olympus DP71, Rungis, France) prior molecular analysis. In addition, a total of 327 louse specimens were also included in this study, corresponding to body and head lice collected from several countries. These samples were obtained from the private frozen collection of world lice belonging to our laboratory. Additional supporting information including, collection locations and numbers of lice tested are described in Table S1.

Moreover, because there were no available 12S sequences of New World monkey louse Pediculus mjobergi in the GenBank database, we have also amplified the $12 \mathrm{~S}$ gene for this louse. In total three $P$. mjobergi lice were amplified in this study targeting $12 \mathrm{~S}$ gene. These samples were obtained from the same P. mjobergi collection, from our laboratory, that was previously used by Drali et al. (Drali et al., 2016). More specifically, the louse specimens were collected from a wild howler monkey, Alouatta caraya, (\#B2188) from the Iguazú National Park, Misiones Province (Drali et al., 2016).

\section{3 DNA extraction, PCR amplification and sequencing}

Genomic DNA was isolated from louse specimens using the DNeasy tissue kit (Qiagen, Courtaboeuf, France) as described previously (Amanzougaghene et al., 2017). Three mitochondrial genes $c y t b, c o x 1$ and $12 \mathrm{~S}$ ribosomal RNA were targeted for sequencing. PCR amplifications were conducted in a Peltier PTC-200 thermal cycler (MJ Research Inc., Watertown, MA, USA) with the Hotstart Taq-polymerase (Qiagen) in accordance with the manufacturer's instructions. The success of PCR amplification was then verified by electrophoresis of the PCR product on a $1.5 \%$ agarose gels. All primers used for these experiments and PCR conditions are described in Table 1. All PCR products were purified using the PCR filter plate Millipore NucleoFast 96 PCR kit (Macherey-Nagel EURL, Hoerdt, France) following the manufacturer's recommendations. The sequence reaction was carried out using the Big Dye Terminator Cycle Sequencing Kit (Perkin Elmer Applied Biosystems, 
151 Foster City, CA) as per the manufacturer's instructions. Sequencing was performed with an 152 ABI Prism 3130xl Genetic Analyzer capillary sequencer (Applied Biosystems). Finally, all 153 the generated sequences were assembled and corrected using ChromasPro 1.7 software 154 (Technelysium Pty Ltd., Tewantin, Australia).

155

156

157 158

\section{4 Sequence analysis.}

All the 98 Amazonian head louse sequences for the three mitochondrial genes (cytb, cox1 and 12S) obtained in this study were combined with all the available mitochondrial sequence data of $P$. humanus (1402 cytb, 743 coxl and $34412 \mathrm{~S})$ from other parts of the world to generate a global dataset to examine clade diversity in $P$. humanus. In addition, the newly amplified $12 \mathrm{~S}$ sequence of $P$. mjobergi in this study, as well as, seven $P$. mjobergi sequences (six cytb and one coxl sequences) reported by Drali et al. (Drali et al., 2016), were also included in the analysis (Table S2).

The DNA sequences obtained from the literature varied in length, so sequences were trimmed to produce a dataset that maximized the number of sequences incorporated. The sequences between nucleotide positions 433-705 of cytb (272-bps, according to Genbank Accession KC685778), 748-1031 of coxl (283-bps, according to Genbank Accession KC685838) and 109-666 of 12S (557-bps, according to Genbank Accession KC685877) were used for analysis. ClustalW alignments were performed in MEGA6 (Tamura et al., 2013). Haplotypes were identified using DnaSP v5.10 software (Librado and Rozas, 2009). Finally, we created three datasets for which a total of 1500,841 and 442 sequences were included, respectively, for cytb, coxl and 12S (Tables S3-S5).

\section{5 Phylogenetic analysis}

Neighbor-joining (NJ) analysis was performed in MEGA6 using the K2P model with pairwise-deletion and 500 bootstrap replicates. The Maximum-likelihood (ML) analysis was also performed in MEGA6 using the Kimura 2-parameter model for nucleotide sequences 
176

177

178

179

180

181

182

183

184

185

186

187

188

189

190

191

192

193

194

195

196

197

198

199

200

under 500 bootstrap replicates. The subtree for each clade of lice was collapsed with the "compress/expand subtree" function. Cytb, coxl and $12 \mathrm{~S}$ sequences from $P$. schaeffi (AY695999, KC241887, AY696067, KC241883 and KR706169) were employed as outgroups.

\section{6 Genetic diversity and haplotype analysis}

For each dataset, population genetic indices including number of sequences (n), number of polymorphic sites $(\mathrm{S})$, average number of pairwise nucleotide differences $(\mathrm{k})$, nucleotide diversity $(\pi)$, number of haplotypes $(\mathrm{H})$ and haplotype diversity $(\mathrm{Hd})$ were calculated using DNASP v5.10 software (Librado and Rozas, 2009). Kimura-2-parameter (K2P) pairwise distances among the cytb, Coxl and 12S haplotypes were calculated using MEGA6 with pairwise deletion of gaps and missing data (Tamura et al., 2013). Neutrality tests (Fu \& Li's D and Tajima's D) were calculated with DNASP v5.10 (Librado and Rozas, 2009). In order to investigate the possible relationships between the haplotypes, networks haplotypes for each of the three genes were constructed with the median joining method of Bandelt available in NETWORK5.0 (www.fluxus-engineering.com/sharenet.htm) using equal weights for all mutations (Bandelt et al., 1999).

\section{Results}

A total of 98 head lice collected from 22 Amazonian individuals were analyzed, targeting three mitochondrial genes (cytb, coxl and 12S). We obtained 11 haplotypes of $c y t b$, 8 haplotypes of coxl and 13 of $12 \mathrm{~S}$ that were defined by 31,25 and 41 polymorphic sites, respectively (Fig. S1-S4). The distribution of the head lice haplotypes identified in this study, according to mitochondrial genes, among the 22 infested Amazonian individuals are presented in Table S6. The generated Amazonian sequences (98 sequences for each gene) were then combined with all available sequences for $c y t b$, coxl and $12 \mathrm{~S}$. The number of haplotypes in each dataset within each clade and their distributions were determined for each 
201

202

203

204

205

206

207

208

209

210

211

212

213

214

215

216

217

218

219

220

221 222 New World monkey louse P. mjobergi, while the remaining 14 of the 98 (14.3\%) Amazonian

223 lice sequences belonged to clade A.

gene. The details of the identified haplotypes, their GenBank accession numbers and geographic locations are described in Tables S3-S5.

In addition, six cytb and one coxl P. mjobergi sequences reported by Drali et al. (Drali et al., 2016), as well as, the three $P$. mjobergi $12 \mathrm{~S}$ sequences amplified in this study were also included in the analysis (Table S2).

For the $c y t b$ dataset, 1506 sequences were included (1500 sequences of $P$. humanus and 6 sequences of $P$. mjobergi) from which 105 haplotypes, including two haplotypes from $P$. mjobergi, were identified from 45 countries on five continents. For the coxl dataset, 842 sequences were included (841 sequences of $P$. humanus and one sequence of $P$. mjobergi) from which 57 haplotypes, including one haplotype from $P$. mjobergi, were identified from 27 countries on five continents. For the $12 \mathrm{~S}$ dataset, 445 sequences were included (442 sequences of $P$. humanus and 3 sequences of $P$. mjobergi) from which 49 haplotypes, including one haplotype from $P$. mjobergi, were identified from 18 countries on five continents.

Neighbor-joining (NJ) and Maximum-likelihood (ML) analyses, including all haplotypes, were performed for each of the three mtDNA genes, consistently recovered six highly divergent and well-supported monophyletic clades (Fig. 2 and Fig. S5). Five clades corresponded to the mitochondrial clades previously recognized A, D, B, C and E, while the sixth was novel, here named "clade F".

This novel clade consists mainly of Amazonian head lice (a total of 84 out of 98 [85.7\%] Amazonian lice sequences belonged to this clade) as well as one haplotype from the lice sequences belonged to clade A.

More precisely, for the $12 \mathrm{~S}$ gene, the clade $\mathrm{F}$ consisted of nine haplotypes; eight haplotypes were for Amazonian lice (referred to here as F19-F26) whose F9 haplotype was 
226 the most common (83.3\% of 84 sequences), while the ninth haplotype was from P. mjobergi.

227 For the coxl gene, 8 haplotypes were identified, six haplotypes were from Amazonian lice 228 (referred to here as F29-F34), one haplotype named F18 consisted of sequences reported by 229 Ascunce et al. (Ascunce et al., 2013) from Argentina (10 coxl sequences) and Mexico (2 230 coxl sequences), the eighth haplotype was $P$. mjobergi. Lastly, for the $c y t b$ gene, the clade $\mathrm{F}$ 231 also included nine haplotypes; eight haplotypes were from Amazonian lice (named here F54 232 and F1-F7) of which haplotype F54 was the most widespread (84.3\% of 89 sequences were 233 from Amazonian head lice sequenced in this study, while $15.7 \%$ of 89 sequences also from 234 Amazonian lice and were recovered from Genbank database, the remaining haplotype was 235 from $P$. mjobergi. It is important to note that the second cytb haplotype of P. mjobergi has the 236 P. humanus' haplotype A5 within clade A (see Drali et al., 2016).

237 The median-joining networks for all cytb, coxl and $12 \mathrm{~S}$ haplotypes corroborated the 238 neighbor joining and Maximum-likelihood phylogenetic reconstructions, with all the 239 recovered haplogroups forming separate clusters represented by six connected subnetworks 240 corresponding to clades A, D, B, C, E and "F" (Fig. 3-5).

241 The maximum intra-clades distances at $c y t b$ were $1.2 \%, 1.9 \%, 1.4 \%, 1.4 \%, 1.5 \%$ and $2421.1 \%$ for clades $A(n=34$ haplotypes), $D(n=17), B(n=9), C(n=13), E(n=23)$ and "F" ( $n=$ 243 8), respectively. The maximum intra-clades distances at coxl were $1.2 \%, 1.0 \%, 1.3 \%, 1.3 \%$, $2441.3 \%$ and $1.1 \%$ for clades $A(n=17), D(n=7), B(n=12), C(n=6), E(n=7)$ and "F" $(n=7)$, 245 respectively. The maximum intra-clades distances at $12 \mathrm{~S}$ were $0.9 \%, 1.6 \%, 0.4 \%, 1.4 \%$, $2460.3 \%$ and $0.5 \%$ for clades $A(n=15), D(n=8), B(n=3), C(n=10), E(n=4)$ and "F" $(n=8)$, 247 respectively. The nearest neighbors $(\mathrm{NN})$ distances between clades and the nodal supports are 248 presented in Fig. 2A (cytb), Fig. 2B (coxl) and Fig. 2C (12S). The new clade "F" shows 249 divergence from its $\mathrm{NN}$ clade B of $5.4 \%, 3.7 \%$ and $3.6 \%$, respectively, at cytb, coxl and 12S. 
252 nucleotide differences (k) and haplotype diversity (Hd) varied among the clades of three 253 genes. The highest haplotype diversity was found within clade D in both $c y t b$ and $12 \mathrm{~S}$ genes $254(\mathrm{Hd}=0.831$ and 0.899 , respectively, in cytb and $12 \mathrm{~S})$, while in coxl gene, the highest 255 haplotype diversity was found within clade B $(\mathrm{Hd}=0.899)$. Overall, both $(\mathrm{k})$ and $(\pi)$ were 256 similar in cytb, coxl and $12 \mathrm{~S}$.

\section{Discussion}

In this study, we analyzed the genetic diversity of head lice collected from Amazonian individuals of the Wayampi community living in Trois-Sauts, a remote and isolated village. In total, three mitochondrial genes were targeted (cytb, coxl and 12S). By coupling these results with all available mitochondrial data of $P$. humanus from 45 countries, the present study has expanded understanding of its levels, sequence divergence pattern and revealed higher levels of mtDNA diversity in $P$. humanus corroborating results reported by others (Ashfaq et al., 2015). To the best of our knowledge, this is the most geographically widespread study to evaluate the mitochondrial genetic diversity in human lice based on three mtDNA genes. Previous studies on $P$. humanus showed that the maximum distances within clades were $1.4 \%$ at $c y t b$ and $1.9 \%$ at coxl, while the $\mathrm{NN}$ distances at these genes were $4.6 \%$ and $2.3 \%$, respectively (Ashfaq et al., 2015). In the present study, the maximum distances within clades were almost similar (cytb 1.9\%, coxl $1.3 \%$ and 1.6\% 12S), while NN distances were higher (cytb 5.6\% and coxl 6.5\%). These results reflect the extended geographic coverage and the large sample sizes, which allowed the recovery of additional clades and haplotypes within each clade.

Three phylogenetic methods (NN, ML and MJ) at three mtDNA genes, have consistently recovered six highly divergent and well-supported monophyletic clades. Five 
275 clades corresponded to the previously recognized mitochondrial clades A, D, B, C and E 276 (Amanzougaghene et al., 2016b; Ashfaq et al., 2015), while the sixth (clade F) was novel, 277 consisting mainly of head lice from Amazonian individuals analyzed in this study and 12 278 sequences of head lice from Argentina and Mexico reported by Ascunce et al. (Ascunce et 279 al., 2013). In that study, the authors found these sequences to be highly derived B-haplotypes 280 that are separated from the main group by more than seven mutational steps (Ascunce et al., 281 2013).

282 Interestingly, the $P$. mjobergi $12 \mathrm{~S}$ and coxl haplotypes, as well as, one of the two $P$. 283 mjobergi cytb haplotypes previously classified within clade B by Drali et al., (Drali et al., 284 2016) belong to this new clade F. This result suggests that $P$. mjobergi may not be a distinct 285 species of $P$. humanus, but probably an evolutionary lineage of $P$. humanus species within 286 clades A and F. A similar suggestion was offered by Drali et al. (Drali et al., 2016). Indeed, 287 these authors argued that $P$. mjobergi was originally a human louse and was switched to new 288 world monkeys from the first humans who reached the American continent thousands of 289 years ago (Drali et al., 2016). Further studies are needed to confirm this hypothesis.

290 Clade "F" shows a divergence from its NN clade B of 5.4\%, 3.7\% and 3.6\%, 291 respectively, at cytb, coxl and $12 \mathrm{~S}$. Clade B was first described in contemporary lice in the 292 American continent, where it was highly prevalent and diversified (Ascunce et al., 2013; 293 Light et al., 2008; Reed et al., 2004). This discovery and its identification in the lice of pre294 Columbian mummies led researchers to deduce initially an American origin for this clade 295 (Boutellis et al., 2013). However, its recent discovery among head lice remains from Israel, 296 dating back about 2,000 years, has challenged this hypothesis (Amanzougaghene et al., 297 2016b). In that study, the authors strongly argued in favor of an Asian origin of clade B, that 298 resulted probably from a recent host switch from Neanderthals or Denisovans to modern 299 humans which was followed by its introduction into the New World with the migration of 
301 et al., 2004). Because clade F was found only in head lice from Mexico and Argentina and it 302 was the dominant lineage found in the Amazonian lice, knowing that Amazonia is one of the 303 few places in the world that has not been strongly affected by globalization. This clade F may 304 be the descendants of a pre-Columbian population and was derived from clade B brought by 305 the first humans who had reached the American continent via the Bering straits thousands of 306 years ago, thus representing Native America louse mitochondrial diversity. Interestingly, 307 Ascunce et al. (Ascunce et al., 2013) have also proposed a similar suggestion for their 308 sequences from Mexico and Argentina that we have reclassified here as members of clade F. Previous studies reported that clade $\mathrm{A}$ is the most common and has a global distribution 310 (Amanzougaghene et al., 2017; Ascunce et al., 2013; Ashfaq et al., 2015), results supported 311 by its detection in approximately $46 \%$ of the analyzed lice from 49 countries on the five 312 continents. Furthermore, the clade A subnetworks at the three analyzed mitochondrial genes 313 (Fig. 3-5) were star-like in structure, combined with its significant negative Tajima's D value $314(-2.322,-1.866$ and -2.055 , respectively at cytb, coxl and $12 \mathrm{~S} ; \mathrm{P}<0.05)$, indicating the 315 signature of population expansion for this clade (Aris-Brosou and Excoffier, 1996), and 316 corroborating the results reported by others (Ascunce et al., 2013; Reed et al., 2004). In 317 addition, Reed et al. (Reed et al., 2004) estimated that the demographic expansion of this 318 clade occurred about 100,000 years ago, coinciding with the out-of-Africa expansion of 319 Homo sapiens, thus reflecting a codemographic pattern between lice and humans (Ascunce et 320 al., 2013; Reed et al., 2004). Clade D (referred as clade E in Ashfaq et al. 2015) diverged 321 from clade A between 0.37 and 0.54 MYa (Ashfaq et al., 2015) and is restricted to Central 322 Africa including DR Congo and Congo-Brazzaville, where it was detected mainly among 323 indigenous Pygmy populations (Amanzougaghene et al., 2016a; Drali et al., 2015). This clade 
324 has also been reported in lice in Ethiopia (Amanzougaghene et al., 2016a) and we identified 325 in this study its occurrence, for the first time, in body lice from Zimbabwe.

326 Our sampling did not encounter any new specimens of clade $\mathrm{C}$, so it remains restricted 327 to Africa and Asia (Amanzougaghene et al., 2016a; Ascunce et al., 2013; Light et al., 2008; 328 Sunantaraporn et al., 2015). Given its early divergence in the Pediculus tree around 2 MYa, 329 this clade may have evolved on archaic hominids in Asia or Africa such as H. erectus (Light 330 et al., 2008; Reed et al., 2004). Lastly, clade E (referred as clade D in Ashfaq et al, 2015) 331 diverged from the MRCA of clade $\mathrm{C}$ between 0.28 and 0.42 MYa (Ashfaq et al., 2015). This 332 clade consists of head lice from West Africa including Senegal and Mali where it was highly 333 prevalent (Amanzougaghene et al., 2017). Its recent detection in the head lice of Nigerian 334 refugees arriving in Algeria and migrant communities living in Bobigny (France) is probably 335 the result of a recent migratory flow from West African countries (Candy et al., 2018; Louni 336 et al., 2018).

\section{Conclusion}

Our study underlines the importance of the use of mitochondrial genes in the analysis 340 of phylogeographic patterns and genetic diversity of $P$. humanus. Six highly divergent and 341 well-supported monophyletic clades were identified. Five clades corresponded to the 342 previously recognized mitochondrial clades A, D, B, C and E, while the sixth "clade F" was 343 novel. The new clade F was found mainly in Amazonia, where it is also shared with the 344 monkey louse $P$. mjobergi and could therefore represent Native American louse 345 mitochondrial diversity.

346 The recovery of additional haplotypes within each of the five clades (A, D, C, E and B)

347 along with the discovery of new clade $\mathrm{F}$, demonstrate that levels of genetic diversity in $P$. 
348 humanus is higher than previously thought, reinforcing the importance of continuing to 349 survey and phylogeographically characterize human lice.

350

351

352

353

354 Acknowledgments

355

The authors thank the Amerindian populations of the Wayampi community living in

356 Trois-Sauts for allowing them to collect the louse specimens.

357 Funding: This study was supported by the Institut Hospitalo-Universitaire (IHU)

358 Méditerranée Infection, the National Research Agency under the program « Investissements

359 d'avenir », reference ANR-10-IAHU-03, the Région Provence-Alpes-Côte d'Azur and the

360 European funding FEDER PRIMI.

361

362

363

364

365

366

367

368

369

370

371 


\section{Figure captions}

380 Figure 1. Map showing the head lice collection site from the Amerindians of the 381 Wayampi community living in Trois-Sauts. A) Geographical localization of louse 382 sampling. B) Amerindian children infested with head lice. C) Human head lice from Trois383 Sauts. $q$ female; $\widehat{o}$ male; nymphs and nits.

384 Figure 2. Neighbor-joining cluster analysis of Cytb (A), Cox1 (B) and 12S (C) 385 haplotypes of $\boldsymbol{P}$. humanus. Bootstrap values (500 replicates) are shown above the branches. 386 The scale bar indicates K2P distances. The node for each clade with multiple haplotypes is 387 collapsed to a vertical triangle, with the horizontal depth indicating the level of intra-clade 388 divergence. Bracketed numbers next to each clade name indicate the number of haplotypes 389 analyzed and the average intra-clade distance. Analyses were conducted in MEGA6

390 Figure 3. Cytb haplotype networks of human body and head lice. Each circle indicates a 391 unique haplotype and variations in circle size are proportional to haplotype frequencies. Pie 392 colors and sizes in circles represent the continents and the number of their sequences for a 393 haplotype. P. mjobergi that belong to the haplotype A5 are included in the portion 394 representing human lice from America.

395 Figure 4. Cox1 haplotype networks of human body and head lice. Each circle indicates a 396 unique haplotype and variations in circle size are proportional to haplotype frequencies. Pie 
397 colors and sizes in circles represent the continents and the number of their sequences for a

398 haplotype

399 Figure 5. 12S haplotype networks of human body and head lice. Each circle indicates a

400 unique haplotype and variations in circle size are proportional to haplotype frequencies. Pie

401 colors and sizes in circles represent the continents and the number of their sequences for a

402 haplotype

403

404 Table 1. Primer sequences used in this study

\begin{tabular}{|c|c|c|c|c|c|}
\hline Target gene & $\begin{array}{l}\text { Primer } \\
\text { name }\end{array}$ & Primer sequences $\left(5^{\prime}-3^{\prime}\right)$ & $\begin{array}{l}\text { Product } \\
\text { size (bp) }\end{array}$ & $\mathrm{Tm}$ & source \\
\hline \multirow[t]{2}{*}{ Cytochrome b } & cytbF & GAGCGACTGTAATTACTAATC & \multirow[t]{2}{*}{348} & \multirow[t]{2}{*}{$56^{\circ} \mathrm{C}$} & \multirow{2}{*}{$\begin{array}{l}\text { (Li et al., } \\
\text { 2010) }\end{array}$} \\
\hline & cytbR & CAACAAAATTATCCGGGTCC & & & \\
\hline \multirow{2}{*}{$\begin{array}{l}\text { Cytochrome } \\
\text { oxidase subunit } 1\end{array}$} & $\operatorname{cox} 1 F$ & GGAGTGAGTTCGATTTTAG & \multirow[t]{2}{*}{828} & \multirow[t]{2}{*}{$55^{\circ} \mathrm{C}$} & \multirow[t]{2}{*}{ This study } \\
\hline & $\operatorname{cox} 1 \mathrm{R}$ & GTGCTGAGGAAAGAAAGTC & & & \\
\hline \multirow{2}{*}{$\begin{array}{l}12 \mathrm{~S} \text { ribosomal } \\
\text { RNA }\end{array}$} & $12 \mathrm{SF}$ & CAGCACTAGCGGTCATACAT & \multirow[t]{2}{*}{596} & \multirow[t]{2}{*}{$56^{\circ} \mathrm{C}$} & \multirow[t]{2}{*}{ This study } \\
\hline & $12 \mathrm{SR}$ & AATGACGGGCGATATGTAC & & & \\
\hline
\end{tabular}

405

406

407

408

409

410

411

412

413

414

415 
423 Table 2. Analysis of genetic diversity indices and neutrality tests (Fu \& Li's D and 424 Tajima's D) on mitochondrial cytb, cox1 and 12S sequences

\begin{tabular}{lllllllll}
\hline & $\mathbf{n}$ & $\mathbf{S}$ & $\mathbf{K}$ & $\boldsymbol{\pi}$ & $\mathbf{h}$ & $\mathbf{H d}$ & $\mathbf{F u} \boldsymbol{\&} \mathbf{L i} \mathbf{s} \mathbf{D}$ & Tajima's D \\
\hline$\underline{\text { Cytb all }}$ & $\underline{\mathbf{1 5 0 0}}$ & 96 & 20.341 & 0.075 & $\underline{\mathbf{1 0 5}}$ & 0.999 & $-2,878(\mathrm{P}<0.05) \mathrm{S}^{*}$ & $-0.172(\mathrm{P}>0.1) \mathrm{NS}$ \\
Clade A & 769 & 33 & 3.098 & 0.011 & 34 & 0.750 & $-3.751(\mathrm{P}<0.02) \mathrm{S}^{* *}$ & $-2.322(\mathrm{P}<0.01) \mathrm{S}^{* *}$ \\
Clade D & 69 & 20 & 5.044 & 0.018 & 17 & 0.831 & $-1.425(\mathrm{P}>0.1) \mathrm{NS}$ & $-0.583(\mathrm{P}>0.1) \mathrm{NS}$ \\
Clade B & 200 & 13 & 3.694 & 0.013 & 9 & 0.789 & $-1.633(\mathrm{P}>0.1) \mathrm{NS}$ & $-1.357(\mathrm{P}>0.1) \mathrm{NS}$ \\
Clade F & 104 & 11 & 2.750 & 0.010 & 9 & 0.700 & $-1.924(\mathrm{P}<0.05) \mathrm{S}^{*}$ & $-1.757(\mathrm{P}<0.05) \mathrm{S}^{*}$ \\
Clade C & 205 & 16 & 3.744 & 0.014 & 13 & 0.792 & $-1.471(\mathrm{P}>0.1) \mathrm{NS}$ & $-1.151(\mathrm{P}>0.1) \mathrm{NS}$ \\
Clade E & 153 & 26 & 4.075 & 0.015 & 23 & 0.803 & $-2.747(\mathrm{P}<0.05) \mathrm{S}^{*}$ & $-1.752(\mathrm{P}>0.05) \mathrm{NS}$ \\
\hline COI all & $\underline{\mathbf{8 4 2}}$ & 67 & 17.255 & 0.061 & $\underline{\mathbf{5 7}}$ & 0.946 & $-0.065(\mathrm{P}>0.1) \mathrm{NS}$ & $0.457(\mathrm{P}>0.1) \mathrm{NS}$ \\
Clade A & 443 & 21 & 3.294 & 0.012 & 17 & 0.769 & $-2.244(\mathrm{P}>0.05) \mathrm{NS}$ & $-1.866(\mathrm{P}<0.05) \mathrm{S} *$ \\
Clade D & 48 & 8 & 2.762 & 0.010 & 7 & 0.739 & $-0.971(\mathrm{P}>0.1) \mathrm{NS}$ & $-1.318(\mathrm{P}>0.1) \mathrm{NS}$ \\
Clade B & 157 & 12 & 3.697 & 0.013 & 12 & 0.786 & $-0.564(\mathrm{P}>0.1) \mathrm{NS}$ & $-0.292(\mathrm{P}>0.1) \mathrm{NS}$ \\
Clade F & 139 & 8 & 2.952 & 0.010 & 8 & 0.738 & $-0.473(\mathrm{P}>0.1) \mathrm{NS}$ & $-0.503(\mathrm{P}>0.1) \mathrm{NS}$ \\
Clade C & 25 & 8 & 3.667 & 0.012 & 6 & 0.782 & $0.457(\mathrm{P}>0.1) \mathrm{NS}$ & $0.274(\mathrm{P}>0.1) \mathrm{NS}$ \\
Clade E & 30 & 8 & 3.524 & 0.012 & 7 & 0.781 & $0.321(\mathrm{P}>0.1) \mathrm{NS}$ & $0.415(\mathrm{P}>0.1) \mathrm{NS}$ \\
\hline$\underline{\mathbf{1 2 S} \text { all }}$ & $\underline{\mathbf{4 4 2}}$ & 110 & 26.928 & 0.048 & $\underline{\mathbf{4 9}}$ & 0.964 & $-0.332(\mathrm{P}>0.1) \mathrm{NS}$ & $-0.130(\mathrm{P}>0.1) \mathrm{NS}$ \\
Clade A & 145 & 30 & 4,886 & 0.009 & 15 & 0.830 & $-2.502(\mathrm{P}<0.05) \mathrm{S}^{*}$ & $-2.055(\mathrm{P}<0.05) \mathrm{S} *$ \\
Clade D & 62 & 23 & 8.821 & 0.016 & 8 & 0.899 & $0.337(\mathrm{P}>0.1) \mathrm{NS}$ & $-0.029(\mathrm{P}>0.1) \mathrm{NS}$ \\
Clade B & 36 & 3 & 0.271 & 0.001 & 3 & 0.679 & $-0.319(\mathrm{P}>0.1) \mathrm{NS}$ & $-1.399(\mathrm{P}>0.1) \mathrm{NS}$ \\
Clade F & 87 & 10 & 2.893 & 0.005 & 9 & 0.746 & $-1.612(\mathrm{P}>0.1) \mathrm{NS}$ & $-1.589(\mathrm{P}>0.05) \mathrm{NS}$ \\
Clade C & 69 & 24 & 7.622 & 0.014 & 10 & 0.885 & $-0.096(\mathrm{P}>0.1) \mathrm{NS}$ & $-0.483(\mathrm{P}>0.1) \mathrm{NS}$ \\
Clade E & 43 & 3 & 1.500 & 0.003 & 4 & 0.606 & $-0.754(\mathrm{P}>0.1) \mathrm{NS}$ & $-0.754(\mathrm{P}>0.1) \mathrm{NS}$ \\
\hline
\end{tabular}


425 n: number of sequences; S: number of polymorphic sites; k: average number of pairwise nucleotide 426 differences; $\pi$ : nucleotide diversity; h: number of haplotypes; Hd: haplotype diversity. Tajima's D: A 427 negative Tajima's D signifies an excess of low frequency polymorphisms relative to expectation. A 428 positive Tajima's D signifies low levels of both low and high frequency polymorphisms. Statistical 429 significance: Not significant, $\mathrm{P}>0.10$.

430

431

432

433

434 


\section{References}

436

437

438

439

440

441

442

443

444

445

446

447

448

449

450

451

452

453

454

455

456

457

458

459

460

461

462

463

464

465

466

467

468

469

470

471

472

473

474

475

476

477

478

479

480

481

Al-Shahrani, S.A., Alajmi, R.A., Ayaad, T.H., Al-Shahrani, M.A., Shaurub, E.-S.H., 2017. Genetic diversity of the human head lice, Pediculus humanus capitis, among primary school girls in Saudi Arabia, with reference to their prevalence. Parasitol. Res. 116, 2637-2643. https://doi.org/10.1007/s00436-017-5570-3

Amanzougaghene, N., Akiana, J., Mongo Ndombe, G., Davoust, B., Nsana, N.S., Parra, H.-J., Fenollar, F., Raoult, D., Mediannikov, O., 2016a. Head Lice of Pygmies Reveal the Presence of Relapsing Fever Borreliae in the Republic of Congo. PLoS Negl. Trop. Dis. 10, e0005142. https://doi.org/10.1371/journal.pntd.0005142

Amanzougaghene, N., Fenollar, F., Sangaré, A.K., Sissoko, M.S., Doumbo, O.K., Raoult, D., Mediannikov, O., 2017. Detection of bacterial pathogens including potential new species in human head lice from Mali. PLOS ONE 12, e0184621. https://doi.org/10.1371/journal.pone.0184621

Amanzougaghene, N., Mumcuoglu, K.Y., Fenollar, F., Alfi, S., Yesilyurt, G., Raoult, D., Mediannikov, O., 2016b. High Ancient Genetic Diversity of Human Lice, Pediculus humanus, from Israel Reveals New Insights into the Origin of Clade B Lice. PloS One 11, e0164659. https://doi.org/10.1371/journal.pone.0164659

Angelakis, E., Diatta, G., Abdissa, A., Trape, J.-F., Mediannikov, O., Richet, H., Raoult, D., 2011. Altitude-dependent Bartonella quintana genotype $\mathrm{C}$ in head lice, Ethiopia. Emerg. Infect. Dis. 17, 2357-2359. https://doi.org/10.3201/eid1712.110453

Aris-Brosou, S., Excoffier, L., 1996. The impact of population expansion and mutation rate heterogeneity on DNA sequence polymorphism. Mol. Biol. Evol. 13, 494-504. https://doi.org/10.1093/oxfordjournals.molbev.a025610

Ascunce, M.S., Fane, J., Kassu, G., Toloza, A.C., Picollo, M.I., González-Oliver, A., Reed, D.L., 2013. Mitochondrial diversity in human head louse populations across the Americas. Am. J. Phys. Anthropol. 152, 118-129. https://doi.org/10.1002/ajpa.22336

Ashfaq, M., Prosser, S., Nasir, S., Masood, M., Ratnasingham, S., Hebert, P.D.N., 2015. High diversity and rapid diversification in the head louse, Pediculus humanus (Pediculidae: Phthiraptera). Sci. Rep. 5. https://doi.org/10.1038/srep14188

Bandelt, H.J., Forster, P., Röhl, A., 1999. Median-joining networks for inferring intraspecific phylogenies. Mol. Biol. Evol. 16, 37-48. https://doi.org/10.1093/oxfordjournals.molbev.a026036

Boutellis, A., Bitam, I., Fekir, K., Mana, N., Raoult, D., 2015. Evidence that clade A and clade B head lice live in sympatry and recombine in Algeria. Med. Vet. Entomol. 29, 94-98. https://doi.org/10.1111/mve.12058

Boutellis, A., Drali, R., Rivera, M.A., Mumcuoglu, K.Y., Raoult, D., 2013. Evidence of sympatry of clade $a$ and clade $B$ head lice in a pre-Columbian Chilean mummy from Camarones. PloS One 8 , e76818. https://doi.org/10.1371/journal.pone.0076818

Candy, K., Amanzougaghene, N., Izri, A., Brun, S., Durand, R., Louni, M., Raoult, D., Fenollar, F., Mediannikov, O., 2018. Molecular Survey of Head and Body Lice, Pediculus humanus, in France. Vector Borne Zoonotic Dis. Larchmt. N. https://doi.org/10.1089/vbz.2017.2206

Chosidow, O., 2000. Scabies and pediculosis. The Lancet 355, 819-826. https://doi.org/10.1016/S0140-6736(99)09458-1

Diatta, G., Mediannikov, O., Sokhna, C., Bassene, H., Socolovschi, C., Ratmanov, P., Fenollar, F., Raoult, D., 2014. Prevalence of Bartonella quintana in patients with fever and head lice from rural areas of Sine-Saloum, Senegal. Am. J. Trop. Med. Hyg. 91, 291-293. https://doi.org/10.4269/ajtmh.13-0685 
Drali, R., Abi-Rached, L., Boutellis, A., Djossou, F., Barker, S.C., Raoult, D., 2016. Host switching of human lice to new world monkeys in South America. Infect. Genet. Evol. J. Mol. Epidemiol. Evol. Genet. Infect. Dis. 39, 225-231. https://doi.org/10.1016/j.meegid.2016.02.008

Drali, R., Shako, J.-C., Davoust, B., Diatta, G., Raoult, D., 2015. A New Clade of African Body and Head Lice Infected by Bartonella quintana and Yersinia pestis-Democratic Republic of the Congo. Am. J. Trop. Med. Hyg. 93, 990-993. https://doi.org/10.4269/ajtmh.14-0686

Durden, L.A., Musser, G.G., 1994. The sucking lice (Insecta, Anoplura) of the world : a taxonomic checklist with records of mammalian hosts and geographical distributions. Bulletin of the AMNH ; no. 218. Sucking lice and hosts.

Goldberger, J., Anderson, J.F., 1912. The Transmission of Typhus Fever, with Especial Reference to Transmission by the Head Louse (Pediculus capitis). Public Health Rep. 1896-1970 27, 297307. https://doi.org/10.2307/4567527

Kim, J.H., Previte, D.J., Yoon, K.S., Murenzi, E., Koehler, J.E., Pittendrigh, B.R., Lee, S.H., Clark, J.M., 2017. Comparison of the proliferation and excretion of Bartonella quintana between body and head lice following oral challenge. Insect Mol. Biol. 26, 266-276. https://doi.org/10.1111/imb.12292

Kittler, R., Kayser, M., Stoneking, M., 2003. Molecular evolution of Pediculus humanus and the origin of clothing. Curr. Biol. CB 13, 1414-1417.

Li, W., Ortiz, G., Fournier, P.-E., Gimenez, G., Reed, D.L., Pittendrigh, B., Raoult, D., 2010. Genotyping of human lice suggests multiple emergencies of body lice from local head louse populations. PLoS Negl. Trop. Dis. 4, e641. https://doi.org/10.1371/journal.pntd.0000641

Librado, P., Rozas, J., 2009. DnaSP v5: a software for comprehensive analysis of DNA polymorphism data. Bioinforma. Oxf. Engl. 25, 1451-1452. https://doi.org/10.1093/bioinformatics/btp187

Light, J.E., Allen, J.M., Long, L.M., Carter, T.E., Barrow, L., Suren, G., Raoult, D., Reed, D.L., 2008. Geographic distributions and origins of human head lice (Pediculus humanus capitis) based on mitochondrial data. J. Parasitol. 94, 1275-1281. https://doi.org/10.1645/GE-1618.1

Light, J.E., Smith, V.S., Allen, J.M., Durden, L.A., Reed, D.L., 2010. Evolutionary history of mammalian sucking lice (Phthiraptera: Anoplura). BMC Evol. Biol. 10, 292. https://doi.org/10.1186/14712148-10-292

Louni, M., Amanzougaghene, N., Mana, N., Fenollar, F., Raoult, D., Bitam, I., Mediannikov, O., 2018. Detection of bacterial pathogens in clade $E$ head lice collected from Niger's refugees in Algeria. Parasit. Vectors 11, 348. https://doi.org/10.1186/s13071-018-2930-5

Murray, E.S., Torrey, S.B., 1975. Virulence of Rickettsia prowazeki for head lice. Ann. N. Y. Acad. Sci. 266, 25-34.

Raoult, D., 2016. A Personal View of How Paleomicrobiology Aids Our Understanding of the Role of Lice in Plague Pandemics. Microbiol. Spectr. 4. https://doi.org/10.1128/microbiolspec.PoH0001-2014

Raoult, D., Reed, D.L., Dittmar, K., Kirchman, J.J., Rolain, J.-M., Guillen, S., Light, J.E., 2008. Molecular identification of lice from pre-Columbian mummies. J. Infect. Dis. 197, 535-543. https://doi.org/10.1086/526520

Raoult, D., Roux, V., 1999. The body louse as a vector of reemerging human diseases. Clin. Infect. Dis. Off. Publ. Infect. Dis. Soc. Am. 29, 888-911. https://doi.org/10.1086/520454

Reed, D.L., Light, J.E., Allen, J.M., Kirchman, J.J., 2007. Pair of lice lost or parasites regained: the evolutionary history of anthropoid primate lice. BMC Biol. 5, 7. https://doi.org/10.1186/1741-7007-5-7

Reed, D.L., Smith, V.S., Hammond, S.L., Rogers, A.R., Clayton, D.H., 2004. Genetic Analysis of Lice Supports Direct Contact between Modern and Archaic Humans. PLOS Biol. 2, e340. https://doi.org/10.1371/journal.pbio.0020340

Sangaré, A.K., Boutellis, A., Drali, R., Socolovschi, C., Barker, S.C., Diatta, G., Rogier, C., Olive, M.-M., Doumbo, O.K., Raoult, D., 2014. Detection of Bartonella quintana in African body and head lice. Am. J. Trop. Med. Hyg. 91, 294-301. https://doi.org/10.4269/ajtmh.13-0707 
Sunantaraporn, S., Sanprasert, V., Pengsakul, T., Phumee, A., Boonserm, R., Tawatsin, A., Thavara, U., Siriyasatien, P., 2015. Molecular survey of the head louse Pediculus humanus capitis in Thailand and its potential role for transmitting Acinetobacter spp. Parasit. Vectors 8, 127. https://doi.org/10.1186/s13071-015-0742-4

Tamura, K., Stecher, G., Peterson, D., Filipski, A., Kumar, S., 2013. MEGA6: Molecular Evolutionary Genetics Analysis Version 6.0. Mol. Biol. Evol. 30, 2725-2729. https://doi.org/10.1093/molbev/mst197

Tishkoff, S.A., Reed, F.A., Friedlaender, F.R., Ehret, C., Ranciaro, A., Froment, A., Hirbo, J.B., Awomoyi, A.A., Bodo, J.-M., Doumbo, O., Ibrahim, M., Juma, A.T., Kotze, M.J., Lema, G., Moore, J.H., Mortensen, H., Nyambo, T.B., Omar, S.A., Powell, K., Pretorius, G.S., Smith, M.W., Thera, M.A., Wambebe, C., Weber, J.L., Williams, S.M., 2009. The Genetic Structure and History of Africans and African Americans. Science 324, 1035-1044. https://doi.org/10.1126/science.1172257

Veracx, A., Raoult, D., 2012. Biology and genetics of human head and body lice. Trends Parasitol. 28, 563-571. https://doi.org/10.1016/j.pt.2012.09.003 
550 Figure S1. (A) Cytb sequences alignment and (B) DNA sequence chromatograms of 551 clade $\mathbf{F}$ haplotypes identified in this study showing the polymorphic sites.

552 Figure S2. (A) Cox1 sequences alignment and (B) DNA sequence chromatograms of 553 clade $\mathbf{F}$ haplotypes identified in this study showing the polymorphic sites.

554 Figure S2. (A) 12S sequences alignment and (B) DNA sequence chromatograms of clade 555 F haplotypes identified in this study showing the polymorphic sites.

556 Figure S4. (A) 12S sequences alignment of clade $F$ haplotypes and (B) 12S DNA 557 sequence chromatogram of $P$. mjobergi amplified in this study.

558 Figure S5. Maximum-likelihood (ML) analysis of Cytb (A), Cox1 (B) and 12S (C) 559 haplotypes of Pediculus humanus. Bootstrap values (500 replicates) are shown above the 560 branches. The scale bar shows K2P distances. The node for each clade with multiple 561 haplotypes is collapsed to a vertical triangle, with the horizontal depth indicating the level of 562 intra-clade divergence. Bracketed numbers next to each clade's name indicate the number of 563 haplotypes analyzed and the average intra-clade distance. Analyses were conducted in 564 MEGA6.

565 Table S1. Additional louse specimens included in this study, obtained from the private 566 frozen collection of world lice belonging to our laboratory.

567 Table S2. Pediculus mjobergi sequences from new world monkey (Alouatta caraya) 568 included in this study. The cox 1 and cytb $P$. mjobergi sequences analyzed in this study were 569 those reported by Drali et al. (2016) collected from two monkey individuals B2188 and 570 B1395. The $12 \mathrm{~S}$ sequences were amplified in this study from three $P$. mjobergi specimens 571 from monkey individual B2188. 
572 Table S3. Geographic occurrences and frequencies of cytb haplotypes of human head 573 and body lice.

574 Table S4. Geographic occurrences and frequencies of cox1 haplotypes of human head 575 and body lice.

576 Table S5. Geographic occurrences and frequencies of 12S haplotypes of human head 577 and body lice.

578 Table S6. Distribution of the head lice haplotypes identified in this study, according to 579 mitochondrial genes, among the 22 infested Amazonian individuals. 


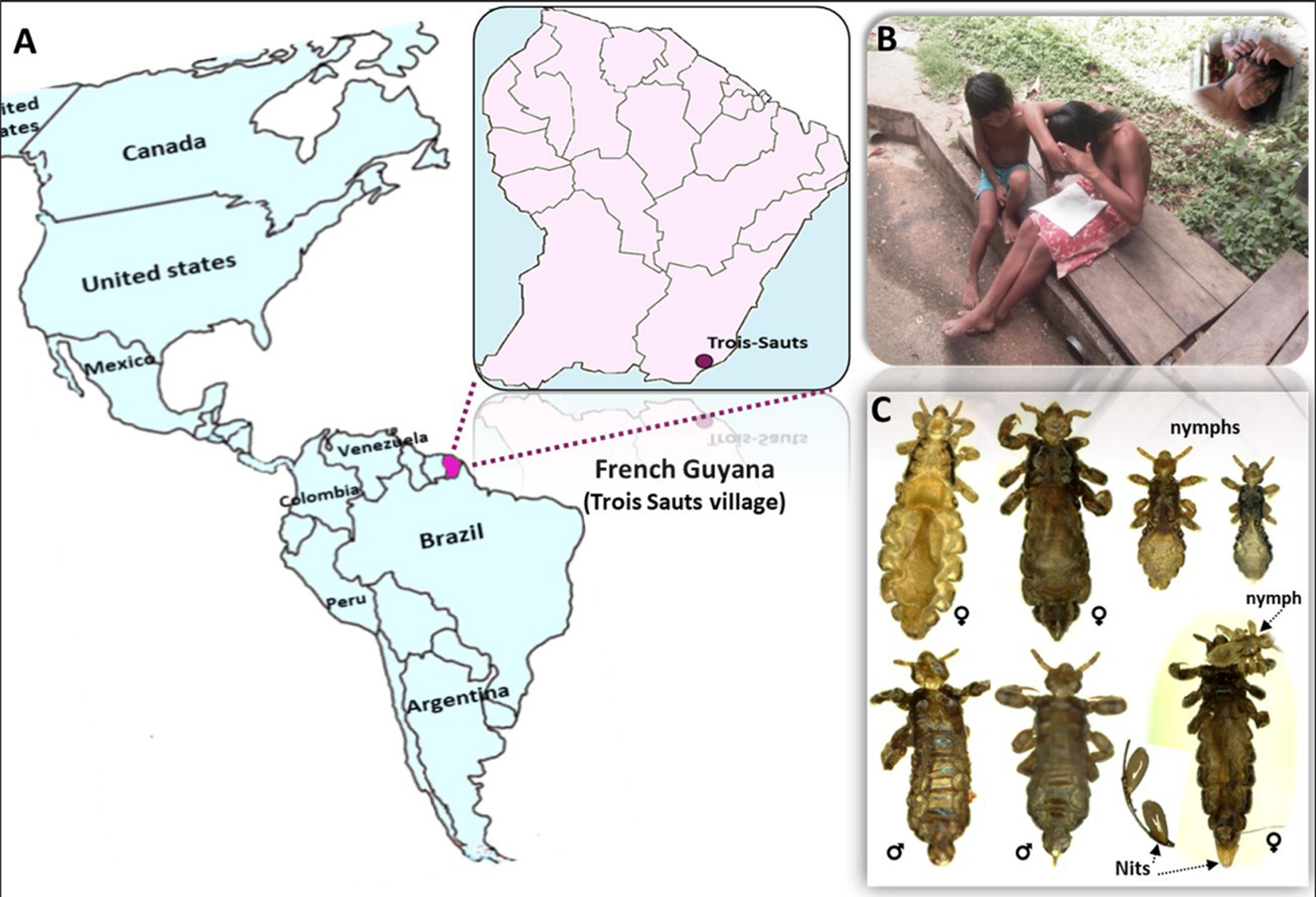




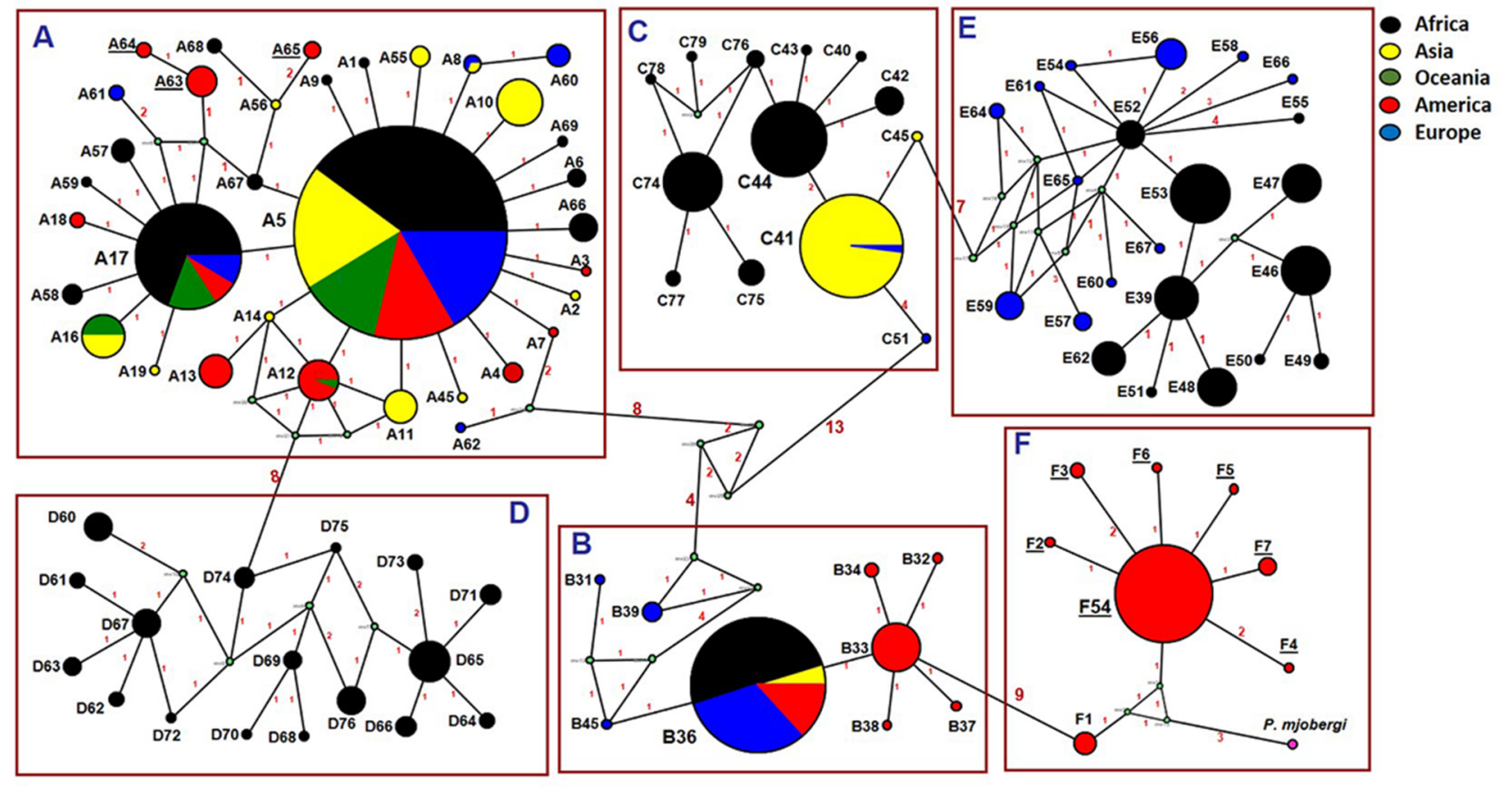




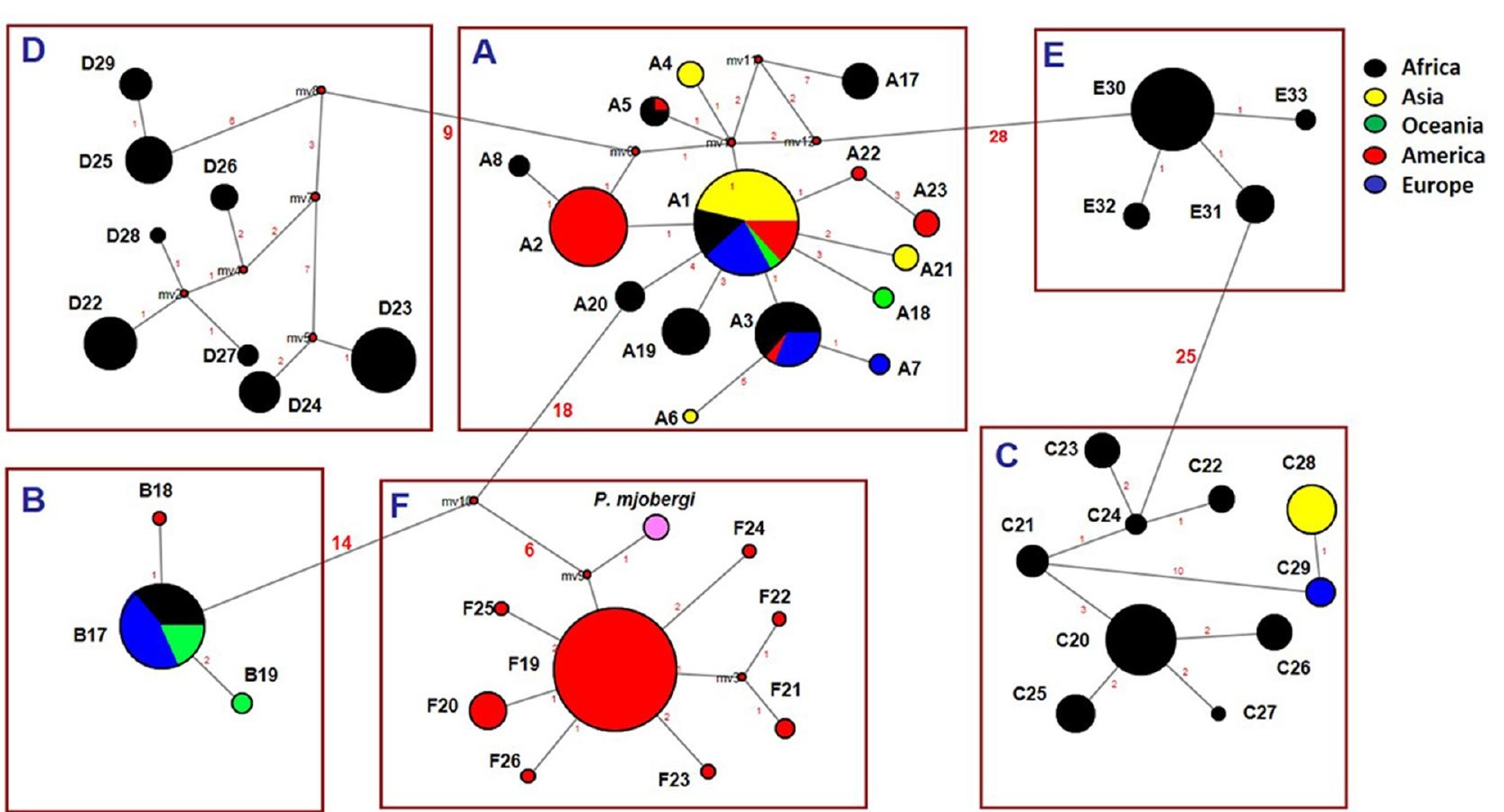




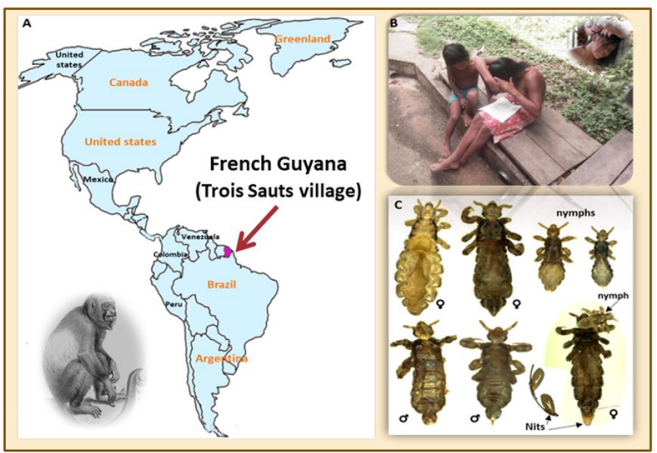

Head lice from an isolated Native American population were analyzed and integrated with all prior data of $P$. humanus from over the world

$\Rightarrow$ New World monkey louse, P. mjobergi, was also included in the analysis
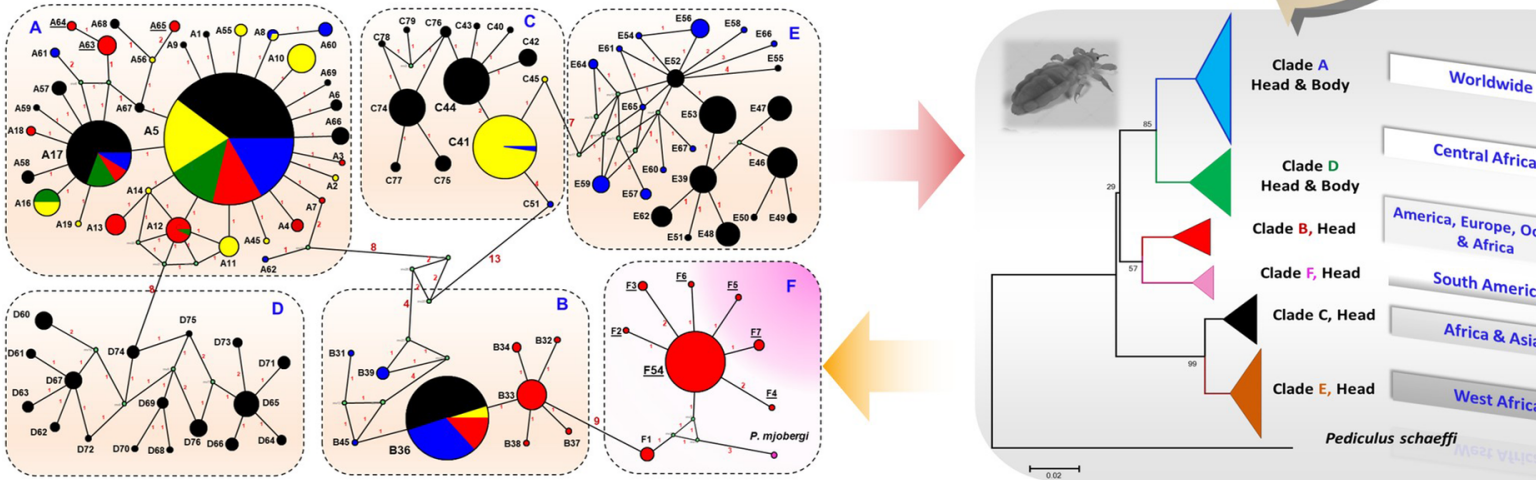

Clade D Head \& Body

Clade B, Head America, Europe, Oceania Clade F, Head

Clade C, Head

South America Africa \& Asia

Clade E, Head West Africa

Pediculus schaeffi 